\title{
Wavelength dependence of the refractive index of human colorectal tissues: comparison between healthy mucosa and cancer
}

\author{
Sónia Carvalho ${ }^{1}$, Nuno Gueiral ${ }^{2,3}$, Elisabete Nogueira ${ }^{2,3}$, Rui Henrique ${ }^{1,4}$, Luís Oliveira ${ }^{2,3^{*}}$, \\ and Valery V. Tuchin ${ }^{5,6,7}$ \\ ${ }^{1}$ Department of Pathology and Cancer Biology and Epigenetics Group-Research Centre, Portuguese Oncology Institute \\ of Porto, Rua Dr. António Bernardino de Almeida S/N, 4200-072 Porto, Portugal \\ ${ }^{2}$ School of Engineering, Physics Department, Polytechnic Institute of Porto, Rua Dr. António Bernardino de Almeida, \\ 431, 4200-072 Porto, Portugal \\ ${ }^{3}$ Centre of Innovation in Engineering and Industrial Technology, ISEP, Rua Dr. António Bernardino de Almeida, 431, \\ 4200-072 Porto, Portugal \\ ${ }^{4}$ Department of Pathology and Molecular Immunology, Institute of Biomedical Sciences Abel Salazar - University of \\ Porto (ICBAS-UP), Rua de Jorge Viterbo Ferreira n228, 4200-072 Porto, Portugal \\ ${ }^{5}$ Research-Education Institute of Optics and Biophotonics, Saratov National Research State University, 83 \\ Astrakhanskaya str., Saratov 410012, Russia \\ ${ }^{6}$ Laboratory of Laser Diagnostics of Technical and Living Systems, Precision Mechanics and Control Institute of the \\ Russian Academy of Sciences, 24 Rabochaya, Saratov 410028, Russia \\ ${ }^{7}$ Interdisciplinary Laboratory of Biophotonics, National Research Tomsk State University, 36 Lenin's av., \\ Tomsk 634050, Russia
}

*e-mail: Imo@isep.ipp.pt

\begin{abstract}
Biological tissues have individual optical properties that may be used as an identity card. The refractive index in particular, which can be measured directly, is very significant for the improvement or development of optical technologies in clinical practice. With the objective of verifying if healthy and pathological tissues can be discriminated from refractive index measurements, we have studied human colorectal mucosa. By using the total internal reflection method to measure the refractive index from healthy and pathological colorectal mucosa tissues at different wavelengths, it was possible to calculate the dispersion curves for both types of tissues. It was observed a decaying refractive index with wavelength, both for healthy and pathological tissues, which were fitted with curves described by Cornu's equation for wavelengths between ultraviolet and near-infrared range. Experimental results show higher refractive index values for the pathological mucosa for all wavelengths. By performing measurements at wavelengths near $850 \mathrm{~nm}$, we have detected non-monotonic behavior for the refractive index of both healthy and pathological tissues. Such abnormal wavelength dependence is evidence of lipids in both tissues. The acquired experimental data demonstrated that it is possible to discriminate between healthy and pathological tissues from refractive index measurements. Similar studies can be made for different biological tissues. By using lasers with different wavelengths it might be possible to identify other tissue components. (C) 2016 Journal of Biomedical Photonics \& Engineering.
\end{abstract}

Keywords: Colorectal tissue, Cancer, Refractive index, Total internal reflection, Tissue dispersion

Paper \#3130 received 2016.12.02; accepted for publication 2016.12.29; published online 2016.12.31. doi: 10.18287/JBPE16.02.040307. [Saratov Fall Meeting 2016 Special Issue]. 


\section{References}

1. J.-C. Lai, Z. Li, C. Wang, and A. He, "Experimental measurement of the refractive index of biological tissues by total internal reflection," Applied Optics 44(10), 1845-1849 (2005).

2. V. V. Tuchin, Tissue Optics: Light Scattering Methods and Instruments for Medical Diagnosis, 3rd Ed., Bellingham, SPIE Press (2015).

3. T. Vo-Dinh, Biomedical Photonics Handbook, CRC Press (2003).

4. P. Giannios, S. Koutsoumpos, K. G. Toutouzas, M. Matiatou, G. C. Zografos, and K. Moutzouris, "Complex refractive index of normal and malignant human colorectal tissue in the visible and near-infrared," J. Biophotonics (2016).

5. F. P. Bolin, L. E. Preuss, R. C. Taylor, and R. J. Ference, "Refractive index of some mammalian tissues using a fiber optic cladding method," Applied Optics 28(12), 2297-2303 (1989).

6. H. Li, and S. Xie, "Measurement method of the refractive index of biotissue by total internal reflection," Applied Optics 35(10), 1793-1795 (1996).

7. Q. Ye, J. Wang, Z.-C. Deng, W.-Y. Zhou, C.-P. Zhang, and J.-G. Tian, "Measurement of the complex refractive index of tissue-mimicking phantoms and biotissue by extended differential total reflection method," J. Biomedical Optics 16(9), 097001-1-5 (2011).

8. J.-C. Lai, Y.-Y. Zhang, Z.-H. Li, H.-J. Jiang, and A.-Z. He, "Complex refractive index measurement of biological tissues by attenuated total reflection ellipsometry," Applied Optics 49(16), 3235-3238 (2010).

9. H. Ding, J. Q. Lu, K. M. Jacobs, and X.-H. Hu, "Determination of refractive indices of porcine skin tissues and intralipid at eight wavelengths between 325 and 1557 nm," J. Opt. Soc. Am. A 22(6), 1151-1157 (2005).

10. Y. L. Jin, J. Y. Chen, L. Xu, and P. N. Wang, "Refractive index measurement for biomaterial samples by total internal reflection," Phys. Med. Biol. 51(20), N271-N379 (2006).

11. J. J. J. Dirckx, L. C. Kuypers, and W. F. Decraemer, "Refractive index of tissue measured with confocal microscopy," J. Biomed. Opt. 10(4), 044014-1-8 (2005).

12. Z. Wang, K. Tangella, A. Balla, and G. Popescu, "Tissue refractive index as marker of disease," J. Biomed. Opt. 16(11), 116017-1-7 (2011).

13. Y. Zhou, K. K. H. Chan, T. Lai, and S. Tang, "Characterizing refractive index and thickness of biological tissues using combined multiphoton microscopy and optical coherence tomography," Biomedical Optics Express 4(1), 38-50 (2013).

14. J. Sun, S. J. Lee, L. Wu, M. Sarntinoranont, and H. Xie, "Refractive index measurement of acute rat brain tissue slices using optical coherence tomography," Optics Express 20(2), 1084-1095 (2012).

15. H. Brenner, M. Kloor, and C. P. Pox, "Colorectal cancer," The Lancet 383(9927), 1490-1502 (2014).

16. S. D. Curran, and L. H. Schwartz, "Colorectal cancer imaging" in Colorectal Cancer: Evidence-based Chemotherapy Strategies, L. B. Saltz (ed.), Humana Press, New Jersey, USA, 219-230 (2007).

17. A. M. Lacy, J. C. García-Valdecasas, S. Delgado, A. Castells, P. Taurá, J. M. Piqué, and J. Visa, "Laparoscopy-assisted colectomy versus open colectomy for treatment of non-metastatic colon cancer: a randomized trial," The Lancet 359(9325), 2224-2229 (2002).

18. R. Subramaniam, A. Mizoguchi, and E. Mizoguchi, "Mechanistic roles of epithelial and immune cell signaling during the development of colitis-associated cancer," Cancer Research Frontiers 2(1), 1-21 (2016).

19. A. Pierangelo, A. Benali, M.-R. Antonelli, T. Novikova, P. Validire, B. Gayet, and A. De Martino, "Ex-vivo characterization of human colon cancer by Mueller polarimetric imaging," Optics Express 19(2), 1582-1593 (2011).

20. J. Wang, Z. Deng, X. Wang, Q. Ye, W. Zhou, J. Mei, C. Zhang, and J. Tian, "Measurement of the refractive index of hemoglobin solutions for a continuous spectral region," Biomedical Optics Express 6(7), 2536-2541 (2015).

21. H. Ding, J. Q. Lu, W. A. Wooden, P. J. Kragel, and X.-H. Hu, "Refractive indices of human skin tissues at eight wavelengths and estimated dispersion relations between 300 and $1600 \mathrm{~nm}$," Phys. Med. Boil. 51(6), 1479-1489 (2006).

22. http://refractiveindex.info/

23. Z. Deng, J. Wang, Q. Ye, T. Sun, W. Zhou, J. Mei, C. Zhang, and J. Tian, "Determination of continuous complex refractive dispersion of biotissue based on internal reflection,” J. Biomed. Opt. 21(1), 015003 (2016).

24. http://omlc.org/spectra/

25. S. Takatani, and M. D. Graham, "Theoretical analysis of diffuse reflectance from a two-layer tissue model," IEEE Trans. Biomed. Eng. 26(12), 656-664 (1979).

26. G. M. Hale, and M. R. Querry, "Optical constants of water in the $200 \mathrm{~nm}$ to $200 \mu \mathrm{m}$ wavelength region," Appl. Opt. 12(3), 555-563 (1973).

27. R. L. P. van Veen, H. J. C. M. Sterenborg, A. Pifferi, A. Torricelli, E. Chikoidze, and R. Cubeddu, "Determination of VIS-NIR absorption coefficients of mammalian fat, with time- and spatially resolved diffuse reflectance and transmission spectroscopy," J. Biomed. Opt. 10(5), 054004-1-6 (2005). 
28. R. Nachabé, B. H. W. Hendriks, M. van der Voort, A. E. Desjardins, and H. J. C. M. Sterenborg, "Estimation of biological chromophores using diffuse optical spectroscopy: benefit of extending the UV-VIS wavelength range to include 1000 to 1600 nm," Optics Express 18(24), 1432-1442 (2010).

\section{Introduction and theoretical background}

In the growing research field of Biomedical Photonics, the knowledge of the refractive index (RI) of biological tissues and fluids is highly necessary to understand and describe how light propagates and interacts with tissue components [1]. There are several diagnosis and treatment applications nowadays that use optical methods [2-3]. These techniques work at different wavelengths within the optical spectral range [2-3]. The $\mathrm{RI}$ is an optical property that depends on the light wavelength and it is characteristic for individual biological materials. Moreover, the RI can be different for healthy and pathological states of the same tissues and may serve as a means for cancer diagnosis, as recently published [4]. Surprisingly, the availability of the RI of biological tissues at different wavelengths is still scarce in literature. There are some methods to measure the RI of biological tissues, but in general, this measurement is made from ex vivo tissue samples. One method consists in using an optical fiber where the tissue serves itself as the fiber cladding [5]. This method allows the calculation of the RI of tissues by considering the total internal reflection of light inside a quartz optical fiber. The authors of reference [5] have measured the RI of various mammalian animal and human tissues. Another method is simply designated by the total internal reflection method, which was proposed in 1996 by Li and Xie to measure the RI of biological tissues [6]. It has been widely used since then and several publications explain how this method works and present results for various wavelengths and tissues. Such method is implemented by placing the biological tissue in contact with the base of a glass prism and illuminating the set with a laser with a particular wavelength through another prism surface. By using polarized laser light as the illuminating beam of the prism/tissue setup at different angles, the amount of reflected light is measured. By knowing the RI of the prism at the laser wavelength, the RI of the tissue can be calculated by identifying the critical angle at the prism/tissue interface [1, 4, 7-10]. This method is very simple and it can be used to measure the RI of biological tissues at different wavelengths, provided that lasers at those wavelengths are available. More recently, imaging methods like confocal microscopy [11], spatial light interference microscopy (SLIM) [12] or optical coherence tomography (OCT) [13-14] have also been proposed to measure the RI of biological tissues.

With such variety of methods to measure the RI of biological tissues, it is now possible to evaluate such parameter for the various biological tissue specimens at different wavelengths. Since the RI is unique for biological tissues and can be used to discriminate between pathological and healthy tissue, we decided to study this optical property at different wavelengths for human colorectal mucosa samples. Our objective was to estimate the dispersion curves of human colorectal mucosa and discriminate between healthy and pathological tissues.

Colorectal carcinoma is the third most common cancer and the fourth cause of cancer-related mortality worldwide. Approximately 1.2 million new cases are diagnosed each year worldwide and $50 \%$ of diagnosed patients will die from the disease [15].

Current technology implies colon endoscopy [16] to establish a reliable diagnosis and colectomy constitutes the basis of surgical treatment, eventually complemented with chemotherapy and/or radiotherapy [17]. Non-invasive optical methods are desired to turn diagnosis/treatment procedures easier and less aggressive to the patient.

Colon and rectum are tube-like structures composed of several layers from the inside to the outside, as represented in figure 1 :

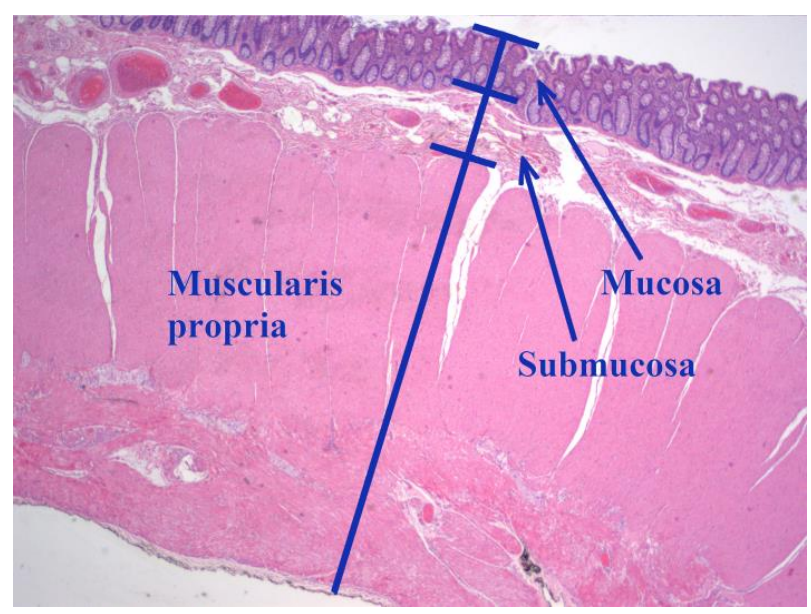

Fig. 1 Structure of the colorectal wall containing histologically distinct layers - from the inside (lumina) outwards: mucosa, submucosa and muscularis propria.

Adenomatous polyps, which are precursor lesions of colorectal carcinoma, originally develop in the innermost colon layer - the mucosa [18]. If polyps are not removed at this early stage, they evolve into adenocarcinoma which intrudes the other layers that compose the colorectal wall, first in the submucosa and eventually reaching the muscularis propria, the subserosal tissue or even the peritoneal lining or adjacent organs [19]. Since the polyps start developing in the mucosa, endoscopic instrumentation can be inserted into the colorectal lúmen to detect them [16] like the one displayed in figure 2: 


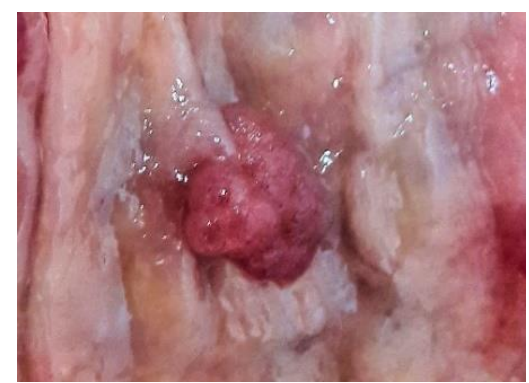

Fig. 2 Colorectal polyp viewed in a surgical specimen.

The polyp in figure 2 shows a more intense red color than the surrounding tissue. Such difference in color is sugestive that it might have higher blood content than its neighbour mucosa tissues. The internal composition of colorectal mucosa and polyps is certainly different and consequently the RI for these tissues will also present different values.

Early this year, a research group has demonstrated that colorectal mucosa and dysplastic colorectal tissues present different RI values at $964 \mathrm{~nm}$ [4]. Since the RI is an optical property that can be measured directly, the discrimination of this parameter between healthy and pathological mucosa at different wavelengths should bring information that can be used for diagnostic and treatment purposes. Considering that optical diagnostic and treatment procedures can operate at different wavelengths, we have studied the RI of colorectal healthy and pathological mucosa samples for visible and near-infrared (NIR) wavelength ranges. This study has produced results that show similar wavelength dependencies for the RI profiles of healthy and pathological mucosa of the human colon. The correspondent dispersion curves were also estimated. The obtained RI values for pathological tissues are higher than the ones obtained for healthy mucosa and evidence of lipid content in both types of tissues was identified.

\section{Materials and methods}

With the objective of measuring the RI of healthy and pathological colorectal mucosa at different wavelengths, we have adopted the total internal reflection method [6]. The preparation of samples and the detailed methodology that was adopted is presented in the following subsections.

\subsection{Tissue samples}

Human colorectal mucosa samples were surgically collected from a population of 5 patients within a 3 month period. These patients were 3 men and 2 women with ages ranging from 52 to 82 . The collected colorectal tissues had large dimensions for our research purposes and contained healthy and pathological areas that could be fragmented into smaller samples to use in our measurements.

A total of 21 healthy and 21 pathological mucosa samples were prepared to be used in our studies.
Healthy and pathological samples were separated and preserved frozen at $-80^{\circ} \mathrm{C}$ for a period of $12-24 \mathrm{hrs}$ prior to the beginning of measurements. A thin slice was made on the top surface of samples to turn them flat so they could be used in measurements with prism. A cryostat (Thermo Scientific ${ }^{\mathrm{TM}}$, model Microm HM 550) was used to make this slice and also to prepare thin samples with $0.4 \mathrm{~mm}$ thickness to use in measurements with Abbe refractometer $(\lambda=589.6 \mathrm{~nm})$. Before studies, samples were kept in saline for $10 \mathrm{~min}$ to mimic natural hydration.

\subsection{Measurement procedure}

The RI measuring setup adopted in this study was the internal reflection method with a dispersion prism as described in literature $[7 ; 20-21]$. Such experimental setup is represented in figure 3 :

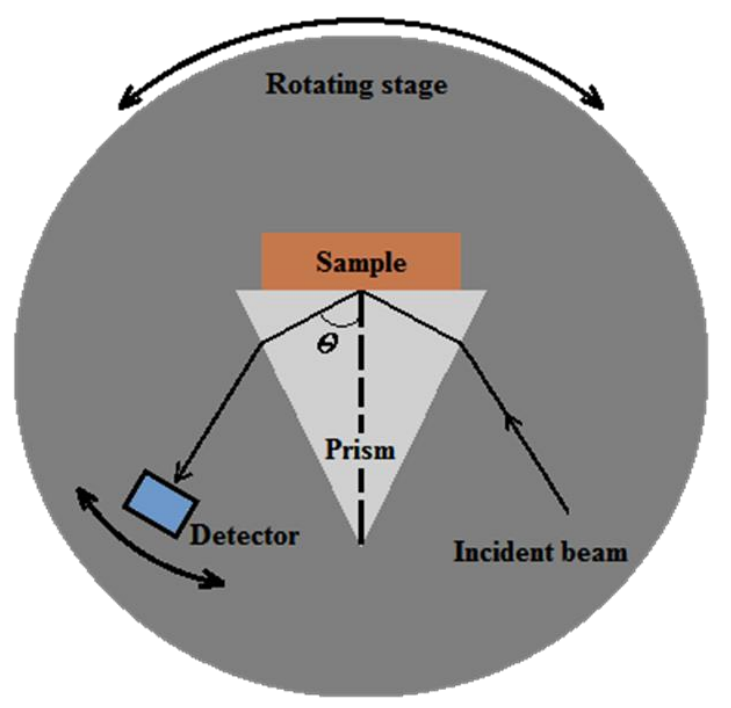

Fig. 3 Internal reflection setup.

Measurements from both types of tissue samples were made by this setup using lasers with wavelengths of 401.4, 532.5, 668.1, 782.1, 820.8 and $850.7 \mathrm{~nm}$. All lasers are laser diodes from Edmund Optics, with the exception for the $668.1 \mathrm{~nm}$, which is from Melles Griot. The measuring prism is a SCHOTT N-SF11 prism and it was also supplied by Edmund Optics. The RIwavelength dependence for this prism is described by the Sellmeier equation [22], Eq. (1), and is represented in figure 4.

$$
n^{2}-1=\frac{K_{1} \lambda^{2}}{\lambda^{2}-L_{1}}+\frac{K_{2} \lambda^{2}}{\lambda^{2}-L_{2}}+\frac{K_{3} \lambda^{2}}{\lambda^{2}-L_{3}}
$$

According to Ref. 22, the Sellmeier coefficients in Eq. (1) take the following values for the SCHOTT NSF11 prism: $\mathrm{K}_{1}=1.7376, \quad \mathrm{~K}_{2}=0.3137, \quad \mathrm{~K}_{3}=1.8988$, $\mathrm{L}_{1}=0.0132, \mathrm{~L}_{2}=0.0623, \mathrm{~L}_{3}=155.2363$. 


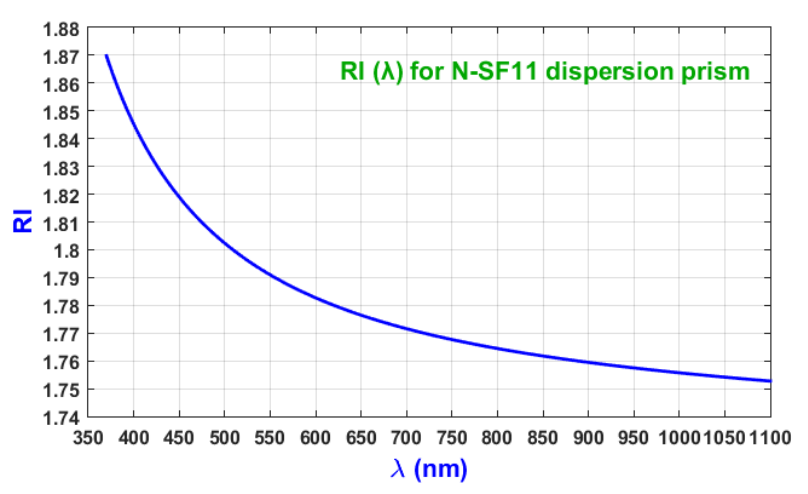

Fig. 4 RI-wavelength dependence for the N-SF11 dispersion prism.

Three sets of measurements were performed with each laser from individual healthy and other three from individual pathological samples to average results. The sequential steps in each individual study were the following:

1. The sample tissue was placed in perfect contact with the base of the prism (see figure 3);

2. An incident laser beam was used to illuminate the setup through a lateral side of the prism;

3. The reflected beam was detected by a photocell connected to a voltmeter to measure the potential difference;

4. This procedure was repeated for several angles of incidence $(\alpha)$ of the beam at the air/prism interface relative to interface normal;

5. Considering Snell-Descartes equation, the incidence angle at the air/prism interface $(\alpha)$ is related to the incidence angle at the prism/tissue interface $(\theta)$ according to:

$$
\theta=\beta-\arcsin \left(\frac{1}{n_{1}} \times \sin (\alpha)\right),
$$

where $\beta$ is the prism internal angle $\left(60^{\circ}\right.$ for our prism) and $n_{1}$ is the RI of the prism at the wavelength of the laser in use.

6. Reflectance at the prism/tissue interface was calculated for each angle as:

$$
R(\theta)=\frac{V(\theta)-V_{\text {noise }}}{V_{\text {laser }}-V_{\text {noise }}}
$$

where $V(\theta)$ is the potential measured at angle $\theta, V_{\text {noise }}$ is the background potential and $V_{\text {laser }}$ is the potential measured directly from the laser.

7. A representation of the reflectance at the prism/tissue interface $(R(\theta))$ as a function of the incident angle $(\theta)$ was created, showing an increase from a lower to a top value as a function of the incidence angle;

8. The first derivative of the previous curve was calculated, showing that a strong peak is observed at a particular incidence angle. This angle is the critical angle $(\theta c)$ of reflection for a particular laser between the prism and the tissue sample;
9. Once $\theta \mathrm{c}$ was determined for a particular laser, it is used to calculate the correspondent RI of the tissue $\left(n_{t}(\lambda)\right)$ at that particular wavelength $(\lambda)$ :

$$
n_{t}(\lambda)=n_{1}(\lambda) \times \sin \left(\theta_{\mathrm{c}}\right) .
$$

In eq. (4), $n_{1}(\lambda)$ represents the RI of the prism at that particular wavelength. Such procedure was made using all lasers mentioned above.

In addition to these measurements, the RI of healthy and pathological mucosa were also measured with the Abbe refractometer $(\lambda=589.6 \mathrm{~nm})$. Three samples of each tissue type (healthy and pathological) were used in these measurements. Since biological tissues are in general turbid, we have prepared these samples with 0.4 $\mathrm{mm}$ thickness and used a $\mathrm{He}-\mathrm{Ne}$ laser to improve contrast when reading the RI from the Abbe refractometer.

After obtaining the RI values of healthy and pathological mucosa for all wavelengths considered in our study, we estimated the dispersion curves for both types of colorectal mucosa tissues. Interpretation of results and discrimination between data for healthy and pathological mucosa was made.

\section{Results and discussion}

As described in the previous subsection, we have performed several sets of measurements from the two types of tissues. For each set of measurements made with a particular laser, the reflectance curve at the prism/tissue interface was calculated. Considering the laser with $\lambda=850.7 \mathrm{~nm}$, figure 5 presents the individual reflectance curves obtained for both types of tissues:

Similar graphs to the ones presented in figure 5 were calculated for the measurements with the other lasers. All graphs in figure 5 show that reflectance increases with the incident angle for both types of tissue, but for the case of pathological mucosa such increase is faster for angles around $50^{\circ}$. The following step consisted on calculating the first derivative of the reflectance curves. To perform this calculation, we have used MATLAB ${ }^{\mathrm{TM}}$. The first derivative is also a function of the incident angle and it was calculated according to equation 5 :

$$
\text { deriv }=\frac{\operatorname{Ref}\left(\theta_{i}\right)-\operatorname{Ref}\left(\theta_{i-1}\right)}{\theta_{i}-\theta_{i-1}}
$$

where $\operatorname{Ref}\left(\theta_{i}\right)$ represents the reflectance at a particular angle, $\theta_{i}$, and $\operatorname{Ref}\left(\theta_{i-1}\right)$ represents the reflectance at the previous angle $\theta_{i-1}$. The derivative calculated with Eq. (5) is the difference between consecutive reflectance values, normalized to the difference between the correspondent angles. This means that Eq. (5) calculates a local derivative. Figure 6 presents the results of these calculations for the datasets presented in figure 5 . 

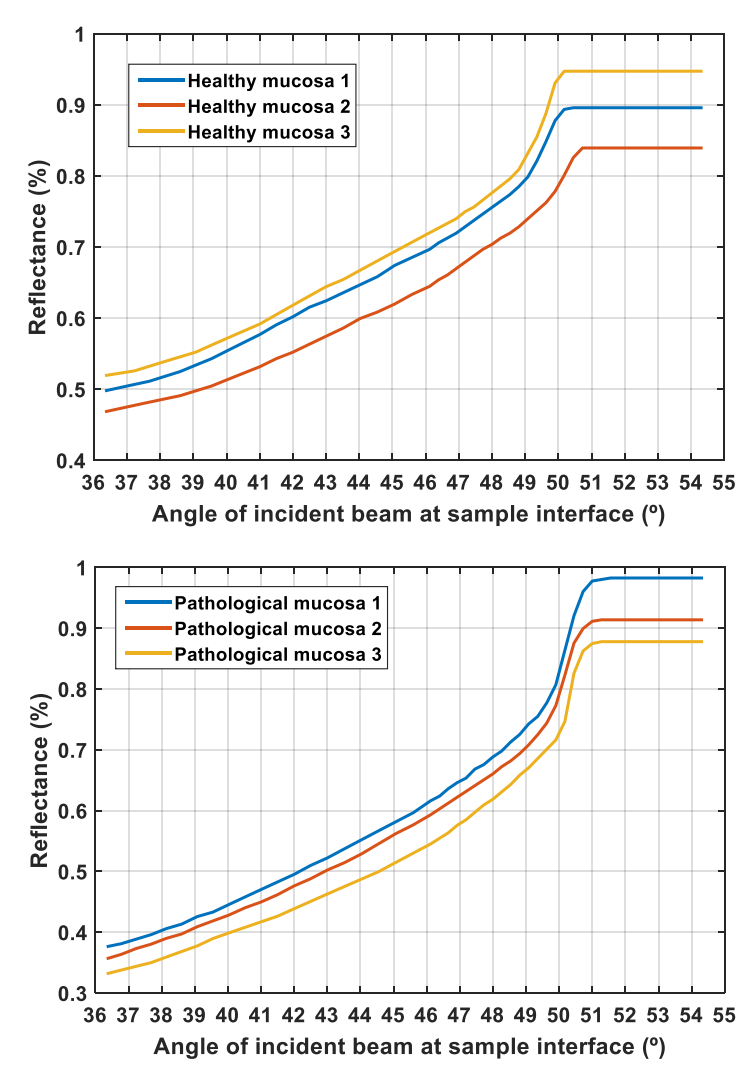

Fig. 5 Individual reflectance curves at the prism/tissue interface for the $850.7 \mathrm{~nm}$ laser.

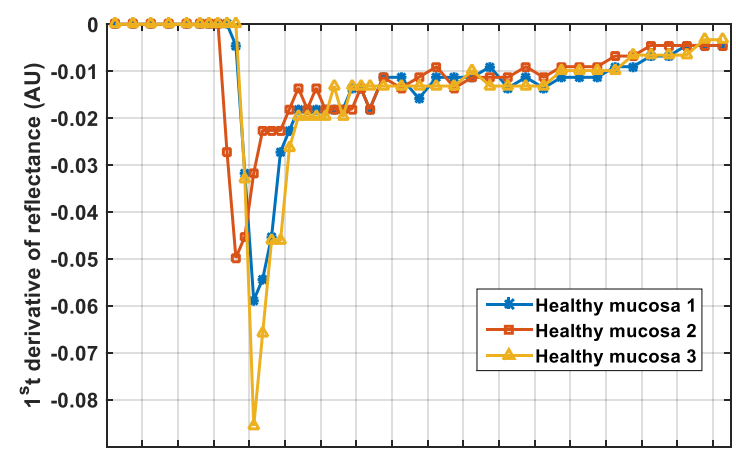

$\begin{array}{llllllllllllllllll}10 & 12 & 14 & 16 & 18 & 20 & 22 & 24 & 26 & 28 & 30 & 32 & 34 & 36 & 38 & 40 & 42 & 44\end{array}$ Angle of incident beam $\left({ }^{\circ}\right)$

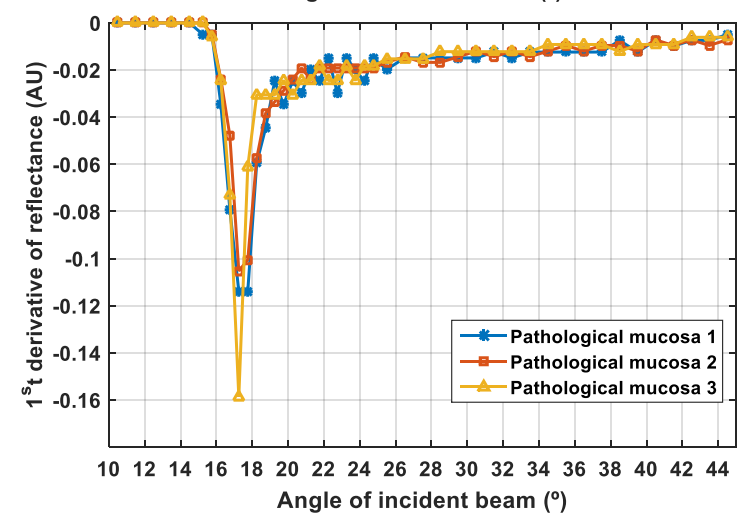

Fig. $61^{\text {st }}$ derivative curves of the reflectance measurements made from colorectal healthy and pathological mucosa samples with the $850.7 \mathrm{~nm}$ laser.
The graphs in figure 6 consider the incident angle at the air/prism interface. Using MATLAB's CFTOOL, each dataset like the ones presented in figure 6 was fitted with a smooth spline curve to estimate the critical angle with precision. The critical angles of reflectance $\left(\alpha_{c}\right)$ correspond to the inverted peaks in previous graphs. A value for the critical angle $\alpha_{\mathrm{c}}$, was obtained for each curve that corresponds to an individual measurement. After obtaining the critical angles for each case, Eq. (2) was used to calculate the correspondent critical angles at the prism/tissue interface, $\theta_{c}$. Using the individual values of $\theta_{\mathrm{c}}$ in Eq. (4) we calculated the RI values for both tissue samples at the various laser wavelengths. Since we performed three studies with each laser, three RI values were obtained for each type of mucosa tissues at every particular laser wavelength. Using these RI values obtained from measurements with same laser, we calculated the mean and SD values at that particular wavelength for healthy and pathological mucosa.

Similarly, mean and SD values were also calculated for the RI of both samples at the wavelength of the Abbe refractometer. All the experimental resulting RI values are presented in table 1 .

The experimental data for healthy mucosa in table 1 is presented in figure 7 along with the calculated dispersion curve indicated in literature [4].

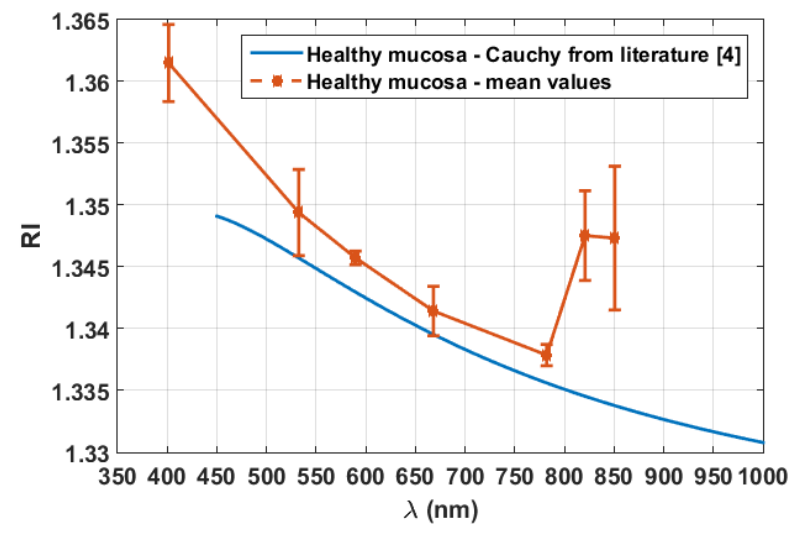

Fig. 7 RI of healthy human colorectal mucosa: experimental and literature data.

The dispersion curve presented in figure 7 was calculated using the following Cauchy equation [4]:

$$
n_{\text {mucosa }}(\lambda)=1.3217+\frac{0.00994}{\lambda^{2}}-\frac{0.00089}{\lambda^{4}} \text {. }
$$

This equation was estimated based on experimental data from 450 to $1550 \mathrm{~nm}$ [4]. To perform calculations with Eq. (6), wavelengths must be considered in $\mu \mathrm{m}$. As we could see from previous figure, for wavelengths between 500 and $800 \mathrm{~nm}$, our experimental results are a little higher than the theoretical curve calculated by the authors of Ref. 4. Wavelength dependence for our experimental RI data is similar to the curve described by Eq. (6) for this range. When considering the uncertainty of the calculated curve, our values are consistent with estimations of the authors of Ref. 4 within the 
Table 1 Measured and calculated mean RI values for healthy and pathological mucosa.

\begin{tabular}{|c|c|c|c|c|c|c|c|}
\hline & \multicolumn{7}{|c|}{$\lambda(\mathrm{nm})$} \\
\hline Healthy mucosa & $\mathbf{4 0 1 . 4}$ & $\mathbf{5 3 2 . 5}$ & $\mathbf{5 8 9 . 6}$ & $\mathbf{6 6 8 . 1}$ & $\mathbf{7 8 2 . 1}$ & $\mathbf{8 2 0 . 8}$ & $\mathbf{8 5 0 . 7}$ \\
\hline Sample 1 & 1.3634 & 1.3510 & 1.3457 & 1.3394 & 1.3377 & 1.3470 & 1.3433 \\
\hline Sample 2 & 1.3579 & 1.3518 & 1.3463 & 1.3415 & 1.3371 & 1.3514 & 1.3540 \\
\hline Sample 3 & 1.3632 & 1.3454 & 1.3452 & 1.3434 & 1.3388 & 1.3442 & 1.3447 \\
\hline Mean & 1.3615 & 1.3494 & 1.3457 & 1.3414 & 1.3379 & 1.3475 & 1.3473 \\
\hline SD & 0.0031 & 0.0035 & 0.0006 & 0.0020 & 0.0009 & 0.0036 & 0.0058 \\
\hline Pathological mucosa & $\mathbf{4 0 1 . 4}$ & $\mathbf{5 3 2 . 5}$ & $\mathbf{5 8 9 . 6}$ & $\mathbf{6 6 8 . 1}$ & $\mathbf{7 8 2 . 1}$ & $\mathbf{8 2 0 . 8}$ & $\mathbf{8 5 0 . 7}$ \\
\hline Sample 1 & 1.3674 & 1.3510 & 1.3508 & 1.3475 & 1.3431 & 1.3581 & 1.3531 \\
\hline Sample 2 & 1.3722 & 1.3475 & 1.3496 & 1.3451 & 1.3427 & 1.3526 & 1.3537 \\
\hline Sample 3 & 1.3690 & 1.3431 & 1.3512 & 1.3451 & 1.3395 & 1.3530 & 1.3559 \\
\hline Mean & 1.3695 & 1.3554 & 1.3505 & 1.3459 & 1.3418 & 1.3546 & 1.3542 \\
\hline SD & 0.0024 & 0.0064 & 0.0008 & 0.0014 & 0.0020 & 0.0031 & 0.0015 \\
\hline
\end{tabular}

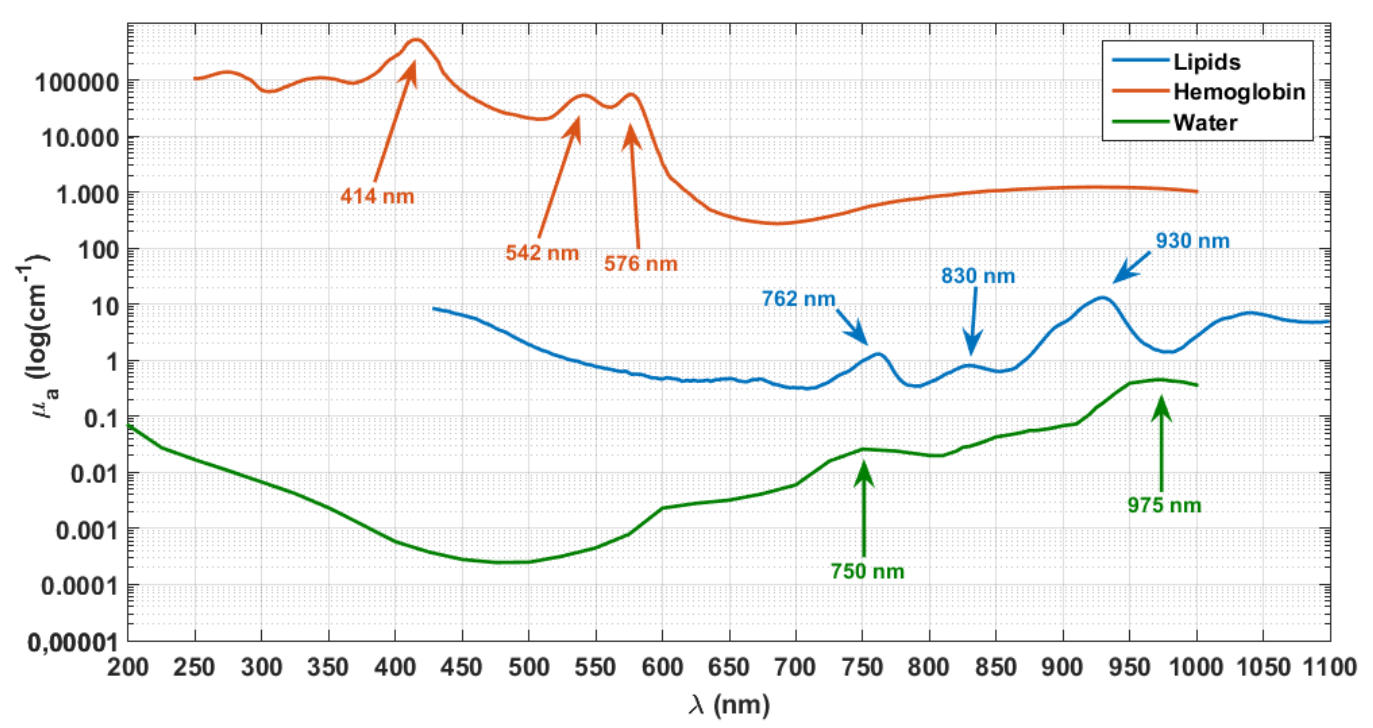

Fig. 8 Absorption coefficient spectra of water, hemoglobin and lipids.

mid-wavelength range. For shorter and longer wavelengths, our experimental results are significantly different from the dispersion curve described by Eq. (6). For ultraviolet wavelengths, our data seems to have more consistent wavelength decay as described in literature $[21,23]$. Such difference is visible, since we have used a laser with $401.4 \mathrm{~nm}$ in our measurements. For longer wavelengths, the difference between our results and the curve described by Eq. (6) is very significant and provides major information. First of all, the authors of Ref. 4 have not obtained measurements between 750 and $950 \mathrm{~nm}$. For this reason, their curve (Eq. (6)), shows very smooth decay in this range. In our case, we see an increase in the RI of mucosa, meaning that some tissue component is responsible for this increase. Searching for an explanation to this anomalous behavior, we have found that lipids contain absorption bands in this spectral range. Figure 8 contains the absorption coefficient spectra for water, hemoglobin and lipids (most common tissue components), as retrieved from the website of the Oregon Medical Laser
Center [24]. Figure 8 is represented in logarithmic scale to contain all data.

According to figure 8, hemoglobin presents a peak at $414 \mathrm{~nm}$ and two others at 542 and $576 \mathrm{~nm}$ [25]. For water we see a peak at $750 \mathrm{~nm}$ and another at $975 \mathrm{~nm}$ [26]. Lipids, on the other hand have three absorption peaks - one at $762 \mathrm{~nm}$, another at $830 \mathrm{~nm}$ and another at $930 \mathrm{~nm}$ [27-28]. When absorption is strong, having similar values to scattering, it is expected to observe an increase in the RI of the sample. From data in figure 8, both water and lipids in our mucosa samples may contribute to the RI increase that we have observed. The water peak at $750 \mathrm{~nm}$ and lipids peak at $762 \mathrm{~nm}$ may also produce a RI increase, but we have not performed measurements within this range of wavelengths. On the other hand, we have measured at 820.8 and at $850.7 \mathrm{~nm}$. At these wavelengths we can see the effect of the second absorption peak of lipids in figure 8. Due to the presence of this peak in this range, absorption and scattering might have a similar value, which originates an increase in mucosa RI. 
Considering our experimental RI data for the lowest wavelengths only (below $820.8 \mathrm{~nm}$ ), we can also estimate a fitting curve for our experimental data. According to literature, there are three equations that are commonly used to fit the RI of biological tissues - the Cauchy equation (equation 6), the Cornu equation (equation 7) and the Conrady equation (equation 8) [21, 23]:

$$
\begin{aligned}
& n_{\text {mucosa }}(\lambda)=A+\frac{B}{\lambda^{2}}+\frac{C}{\lambda^{4}}, \\
& n_{\text {mucosa }}(\lambda)=A+\frac{B}{(\lambda-C)^{\prime}}, \\
& n_{\text {mucosa }}(\lambda)=A+\frac{B}{\lambda}+\frac{C}{\lambda^{3.5}} .
\end{aligned}
$$

Using MATLAB's CFTOOL, we have tried to fit our RI data with these equations. Considering RI data for healthy mucosa, we have obtained best results when fitting with Cornu and Conrady equations (Eqs. (8) and (9)). The fitting with Cauchy equation was poor, giving an R-square value of 0.356 . On the other hand, when using Cornu's or Conrady's equations, we have obtained R-square values of 0.999 in both fittings. Since fitting quality is the same for the two equations, we have selected Cornu's equation to fit our data. Figure 9 shows our experimental data and the calculated fitting curve:

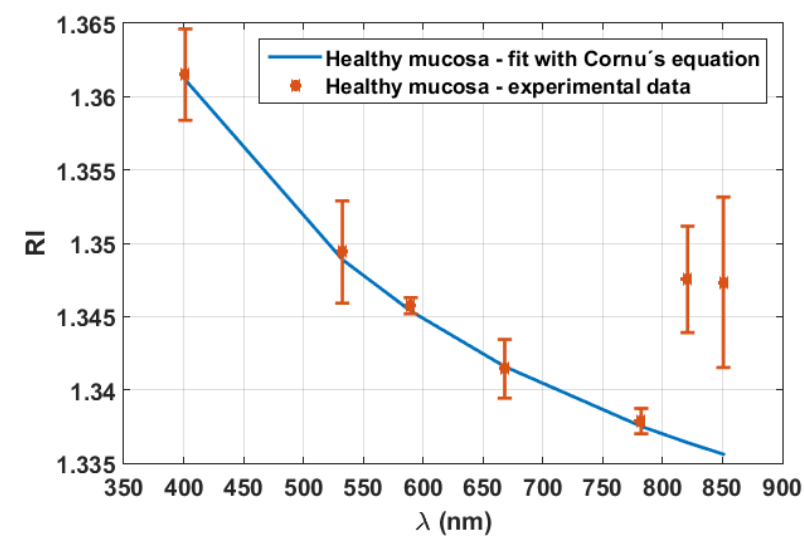

Fig. 9 Healthy mucosa - experimental data and fit curve.

As can be seen from figure 9, when calculating the fitting curve for the RI of healthy mucosa, we have neglected the experimental points at 820.8 and 850.7 nm.

The numerical parameters in the Cornu equation (Eq. (8)) that we have for this fitting are the ones in Eq. (10):

$$
n_{\text {mucosa }}(\lambda)=1.315+\frac{16.73}{(\lambda-38.84)}
$$

Performing a similar fitting to the RI data for the pathological mucosa, we have also obtained best fitting with Cornu and Conrady equations (Eqs. (7) and (8)). Once again selecting Cornu's equation, we have obtained the fitting curve represented in figure 10 and in equation 11:

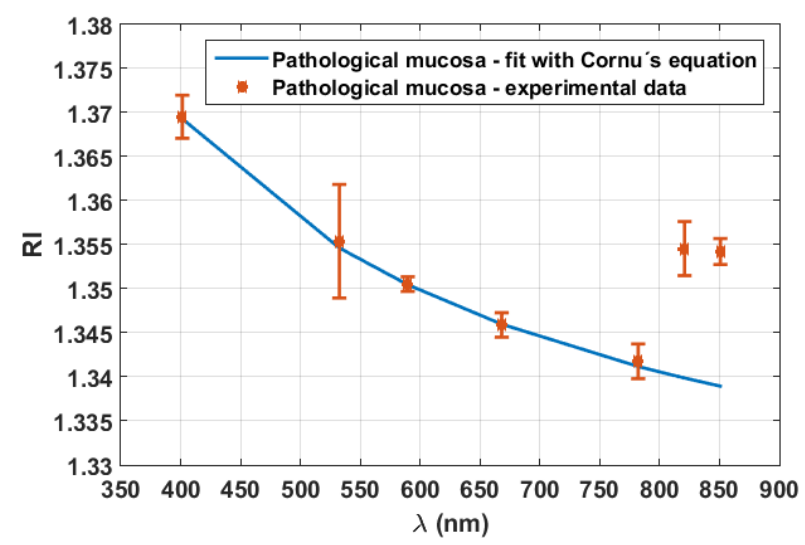

Fig. 10 Pathological mucosa - experimental data and fit curve.

$$
n_{\text {mucosa }}(\lambda)=1.315+\frac{19.25}{(\lambda-46.83)}
$$

As we have demonstrated, the best fittings for the decreasing behavior of RI with wavelength for healthy and pathological mucosa are obtained using Cornu's and Conrady's equations. Since both equations provide R-square values of 0.999 , we have selected Cornu's equation to fit our experimental data.

From this analysis, we see that healthy and pathological mucosa show the same type of behavior for the RI as a function of wavelength. We have also seen that selecting the appropriate laser wavelengths it is possible to use the total internal reflection method to identify the presence of certain tissue components. In our case, by measuring at 820.8 and $850.7 \mathrm{~nm}$, we could identify the presence of lipids in both types of tissues. Considering figure 8 , it is possible that the other lipid peaks $(762$ and $930 \mathrm{~nm})$ can be detected, provided that we perform measurements at those wavelengths in future. Regarding longer wavelengths and considering the data presented in Ref. 4, we assume that the decaying behavior of the RI of healthy and pathological mucosa is maintained.

\section{Conclusions and future perspectives}

Using the total internal reflection method, we have measured the RI values from healthy and pathological mucosa tissues at different wavelengths between the ultraviolet and near-infrared. Using the experimental data, we could estimate the dispersion curves for the 
two types of tissues. Considering the visible wavelength range, our experimental data is much approximated to data presented in literature [4]. Since the lowest laser wavelength we used in our measurements was 401.4 nm, we have verified that both Cornu and Conrady equations are the best to fit the decaying RI behavior with wavelength that is described in literature for biological tissues [21,23]. Cauchy's equation allows for good RI fittings for wavelengths above $450 \mathrm{~nm}$, as demonstrated by authors of Ref.4, but as we have observed it provides poor fitting for smaller wavelengths.

We have obtained the same decaying behaviur for the RI of both colorectal tissues, but pathological mucosa presents higher RI values than healthy mucosa in the entire range of wavelengths. Such difference is significantly important for establishing diagnosis/treatment procedures that use optical technologies. By measuring at 820.8 and $850.7 \mathrm{~nm}$, we were able to identify the presence of lipids in both tissues through the non-monotonic behavior observed. Lipids have strong absorption bands in this range of spectra as presented in figure 8 and the similar levels of scattering and absorption coefficients originate an increase in the RI. In the near future we intend to perform similar measurements for other wavelengths, expecting to identify other tissue components. We will also perform similar measurements for other biological tissues to obtain their dispersion curves. Such data is of high importance for the improvement and development of current and future technologies to use in clinical practice.

Another way to discriminate between healthy and pathological tissue is to estimate their optical properties. To obtain these optical properties, we need to perform inverse simulations, using the RI and other optical measurements. We plan to perform also this study in the near future for colorectal mucosa tissues.

\section{Acknowledgments}

The authors appreciate the availability of instrumental and technical resources made available by I3S-Porto and the help of Cláudia Machado in preparing the tissue samples used in these studies.

This research was supported by the Portuguese research grant FCT-UID/EQU/00305/2013.

VVT was supported by the Russian Presidential grant NSh-7898.2016.2, the Russian Governmental grant 14.Z50.31.0004, and The National Research Tomsk State University Academic D. I. Mendeleev Fund Program. 